\title{
New Method for Determination of Hydrogen Abstraction Ability of Organic Peroxides
}

\author{
Yasumasa Watanabe, ${ }^{\dagger}$ Hideyo IshIGaKi, Hiroshi OKada, \\ and Shuji Suyama
}

Fine Chemicals \& Polymers Research Laboratory, NOF Corporation, Taketoyo-cho, Chita-gun, Aichi 470-23, Japan

(Received April 30, 1997)

\begin{abstract}
The hydrogen abstraction ability of dialkyl peroxides was determined in the presence of $2.0 \mathrm{M} 2,4-$ diphenyl4-methyl-1-pentene ( $\alpha$-methylstyrene dimer, MSD) in cyclohexane at $140^{\circ} \mathrm{C}$. Cyclohexyl radicals produced through hydrogen abstraction by peroxide-derived radicals are efficiently trapped by MSD. Trapping yields were greatly dependent on individual peroxides, decreasing in the following order: di- $t$-butyl peroxide $(72 \%)>$ dicumyl peroxide $(54 \%)>$ di- $t$-amyl peroxide $(22 \%)>$ di- $t$-hexyl peroxide $(14 \%)$. In this case, the main hydrogen abstracting radicals were alkoxy radicals produced from the peroxides. The hydrogen abstraction ability of dialkyl peroxides was also tested in neat cyclohexane. The dehydrodimer yields of cyclohexane were almost the same $(40-50 \%)$ and close to theoretical values. This clearly demonstrates that alkyl as well as alkoxy radicals act as effective hydrogen abstractors in the absence of MSD. MSD trapping is useful for providing information on hydrogen abstraction ability of organic peroxides in the presence of styrenic monomers.

KEY WORDS Radical Trapping / $\alpha$-Methylstyrene Dimer / Addition-Fragmentation / Hydrogen Abstraction / Dialkyl Peroxides /
\end{abstract}

Organic peroxides are industrially useful as initiators for free-radical crosslinking and grafting reactions as well as initiators for the polymerization of vinyl monomers. Crosslinking reactions such as crosslinking of polyolefins and grafting reactions such as graft polymerization of styrene onto polyolefins are induced by hydrogen abstraction ( $\mathrm{H}$-abstraction) by free radicals generated from organic peroxides. Therefore, $\mathrm{H}$-abstraction ability of organic peroxides plays an important role in these applications.

There are two main factors determining the $\mathrm{H}$-abstraction ability of organic peroxides, i.e., free radical production efficiency of peroxides and $\mathrm{H}$-abstraction ability of peroxide-derived radicals. The most convenient method for evaluating $\mathrm{H}$-abstraction ability is to measure the yields of dehydrodimers of solvents after complete decomposition of peroxides in solvents such as $n$-alkanes. ${ }^{1}$ This method (dimer method) is the model reaction of crosslinking reactions. The data obtained by the dimer method can be closely related to the crosslinking efficiency of polymers by peroxides when solvents are suitable model compounds of the desired polymers.

Compared to crosslinking reactions, grafting reactions are more complicated since both vinyl monomers and matrix polymers coexist. In this case, $\mathrm{H}$-abstraction from polymers competes with addition to monomers. That is, some free radicals generated from peroxides are directly trapped by monomers before abstracting hydrogens from matrix polymers. To understand the mechanisms of grafting reactions and select the most suitable organic peroxides for grafting reactions, $\mathrm{H}$-abstraction data of organic peroxides in the presence of monomers or monomer-like compounds are quite helpful. There is no detailed information on such $\mathrm{H}$-abstraction mainly because of the lack of a suitable method of evaluation.

Recently, we developed a new radical trapping tech-

$\dagger$ To whom all correspondence should be addressed. nique using 2,4-diphenyl-4-methyl-1-pentene ( $\alpha$-methylstyrene dimer, MSD) as a radical trapping agent. ${ }^{2}$ This technique is based on the unique radical chemistry of MSD, that is, addition-fragmentation reactions between free radicals and MSD (eq 1 and 2 ). ${ }^{3}$ The easy deactivation of cumyl radicals by self-reactions (eq 3 ) is also a factor.

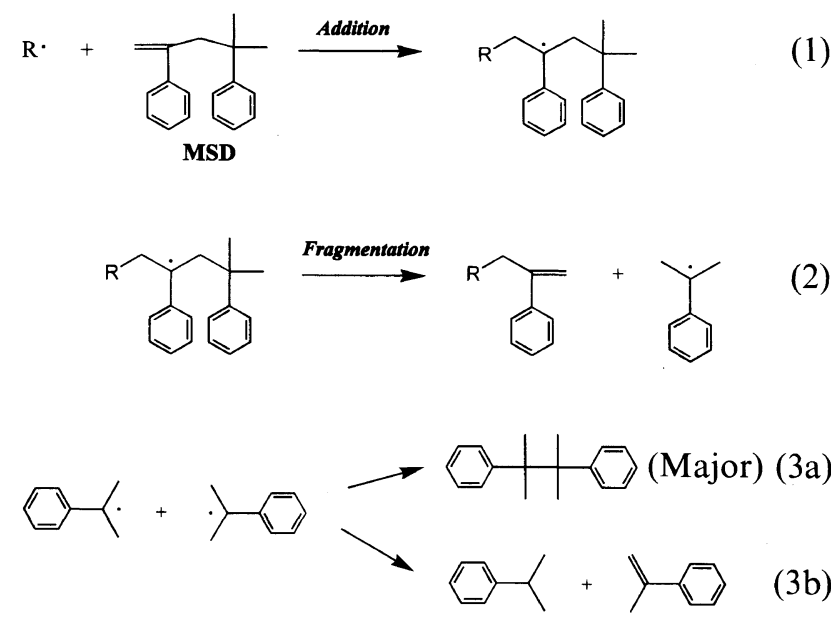

MSD trapping is outlined in Scheme 1. When organic peroxides decompose in MSD, olefinic compounds (1) having various fragments $(\mathrm{X})$ derived from organic peroxides are produced through addition-fragmentation reaction. By analyzing the trapping products (1), we obtain useful information on the free radical chemistry of organic peroxides. We demonstrated that the double bond reactivity of MSD is about the same as that of styrene. ${ }^{2}$ Therefore, the trapping products are closely related to initiating radicals in styrene polymerization.

This technique should be useful for assessing $\mathrm{H}$-abstraction ability of organic peroxides in various $\mathrm{H}$-donor substrates in the presence of styrenic monomers. When organic peroxides are decomposed in the mixture of $\mathrm{H}$ - 
Organic Peroxides

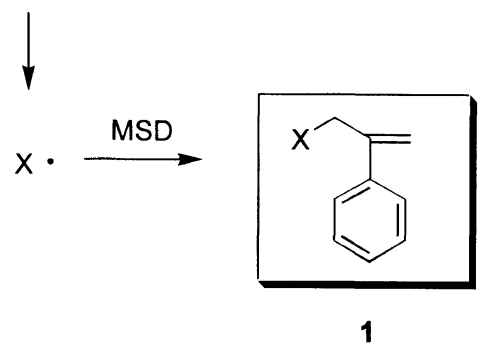

Scheme 1. Outline of MSD trapping.

Organic Peroxides

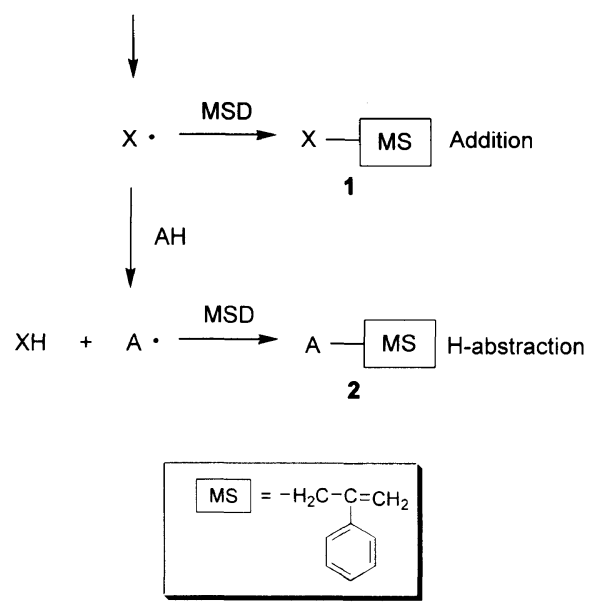

Scheme 2. Method for evaluation of H-abstraction ability of organic peroxides.

donor substrates $(\mathrm{AH})$ and MSD, some peroxide-derived radicals are directly trapped by MSD, while some abstract hydrogens from substrates giving trapping products (2) (Scheme 2). Thus, the yields of $\mathbf{2}$ should reflect $\mathrm{H}$-abstraction ability of organic peroxides in the presence of styrenic monomers.

Dialkyl peroxides are most frequently used as initiators for grafting reactions. However, $\mathrm{H}$-abstraction ability in the presence of monomers has not been presented. This paper describes $\mathrm{H}$-abstraction ability of four dialkyl peroxides (3) in cyclohexane in the presence of MSD at $140^{\circ} \mathrm{C}$. H-abstraction ability was found to greatly depend on the peroxide structure. H-Abstraction ability data are compared with those obtained by the dimer method in neat cyclohexane.

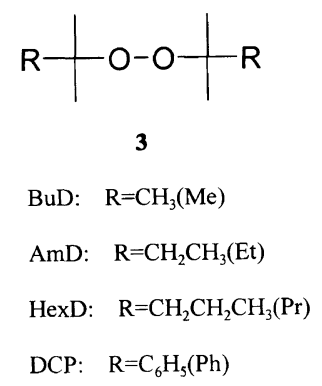

\section{EXPERIMENTAL}

\section{Measurements}

GLC analysis was performed with Shimadzu GC-14A and GC-17A gas chromatographs with a flame ionization detector using capillary columns $(15 \mathrm{~m} \times 0.53 \mathrm{~mm}$ or $25 \mathrm{~m} \times 0.25 \mathrm{~mm}$ ). GC-MS analysis was conducted on a Shimadzu QP5000 under electron impact conditions $(70 \mathrm{eV})$.

\section{Materials}

MSD was obtained from NOF Corporation (Nofmer MSD) and used after purifying by distillation. Di- $t$-butyl peroxide $(\mathrm{BuD}, 99.2 \%$ purity) and dicumyl peroxide (DCP, $99.9 \%$ purity) were obtained from NOF Corporation and used as received. Di- $t$-amyl peroxide (AmD) and di-t $t$-hexyl peroxide (HexD) were synthesized and the methods were described previously. ${ }^{2}$ Used cyclohexane was guaranteed reagent grade.

\section{MSD Trapping Experiments}

An aliquot $(2 \mathrm{ml})$ of a $0.02 \mathrm{M}$ solution of dialkyl peroxides (3) in a mixture of cyclohexane (4.7 M) and MSD $(2.0 \mathrm{M})$ was charged into a glass ampoule. The ampoule was purged with nitrogen, sealed, and immersed in a constant temperature bath at $140^{\circ} \mathrm{C}$ for $15 \mathrm{~h}$. The reaction products were analyzed by GLC and GC-MS. Typical conditions of GLC analysis were: column, $25 \mathrm{~m} \times$ $0.25 \mathrm{~mm}$ capillary column of silicon OV-1 (Shimadzu, HR-1); injector temperature, $150^{\circ} \mathrm{C}$; detector temperature, $250^{\circ} \mathrm{C}$; initial oven temperature, $50^{\circ} \mathrm{C}$; initial hold, $10 \mathrm{~min}$; program rate $1,10^{\circ} \mathrm{C} \mathrm{min}{ }^{-1}$; middle oven temperature, $150^{\circ} \mathrm{C}$; middle hold, $10 \mathrm{~min}$; program rate 2 , $10^{\circ} \mathrm{C} \mathrm{min}^{-1}$; final oven temperature, $250^{\circ} \mathrm{C}$; final hold, 10 min; carrier gas, $\mathrm{He}\left(0.4 \mathrm{ml} \mathrm{min}^{-1}\right)$; split ratio, $1: 18$.

The data for peroxide-derived trapping products were described previously. ${ }^{2} \mathrm{GC}$ retention time and MS spectra of cyclohexyl radical trapping product (2a) are given below. The MS spectra showed the parent peak and characteristic fragment peaks of $117,103,91$, and 77 due to the 2-phenylallyl group of MSD.

3-Cyclohexyl-2-phenyl-1-propene (2a). GC retention time, $36.1 \mathrm{~min}$; MS $(70 \mathrm{eV}) \mathrm{m} / \mathrm{z} 200\left(\mathrm{M}^{+}\right), 185,157,143$, $118,117,103,91,77,55$, and 41.

\section{RESULTS AND DISCUSSION}

\section{MSD Trapping}

The thermal decomposition of dialkyl peroxides (3) was carried out in a mixture of cyclohexane $(4.7 \mathrm{M})$ and MSD $(2.0 \mathrm{M})$ at $140^{\circ} \mathrm{C}$ for $15 \mathrm{~h}$ under nitrogen. Decomposition percentages were over $99.9 \%$. Reaction products were confirmed by GLC and GC-MS. The trapping of cyclohexyl and peroxide-derived radicals was observed. GLC yields of trapping products based on total radicals produced theoretically from peroxides ( 2 mol of radicals per mol of 3 ) and possible schemes leading to their formation are depicted in Schemes 3-6. Total trapping yields were $92,98,87$, and $102 \%$ for $\mathrm{BuD}$, AmD, HexD, and DCP, respectively, indicating high radical recovery in all cases.

The yields of the cyclohexyl trapping product (2a) were greatly dependent on individual peroxides, i.e., 72, 22,14 , and $54 \%$ for $\mathrm{BuD}, \mathrm{AmD}, \mathrm{HexD}$, and $\mathrm{DCP}$, respectively. Thus, $\mathrm{H}$-abstraction ability of dialkyl peroxides greatly differs in the presence of monomer-like compounds. In addition to radical trapping products, 
the formation of $t$-alcohol derived from alkoxy moiety of 3 was confirmed. The yields of alcohol were almost identical with those of $\mathbf{2 a}$. The yield of cumyl alcohol was $55.3 \%$ in the case of DCP $(2 \mathbf{a}=54.3 \%)$. H-Abstraction thus occurs mainly or exclusively by alkoxy radicals under the present conditions. Alkyl radicals such as methyl radicals are rapidly trapped by MSD before participating in $\mathrm{H}$-abstraction from cyclohexane. Alkyl radicals are much less reactive in $\mathrm{H}$-abstraction than alkoxy radicals. Carrock and $\mathrm{Szwarc}^{4}$ reported that the rate of addition of methyl radicals to styrene is about 800 times that of $\mathrm{H}$-abstraction by methyl radicals from isooctane at $65^{\circ} \mathrm{C}$. The rate constant for $\mathrm{H}$-abstrac-

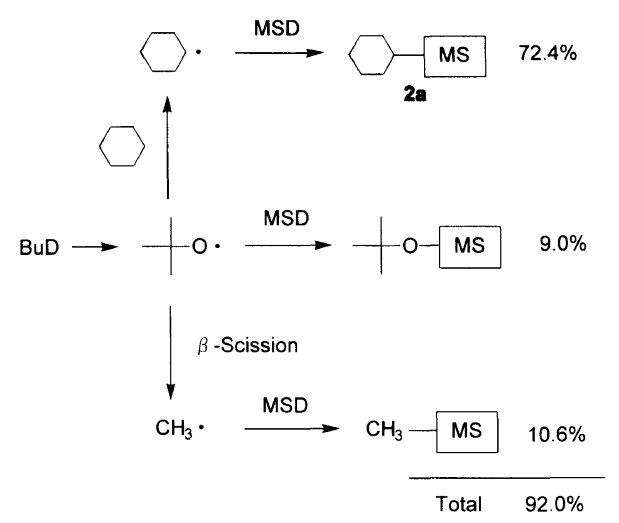

Scheme 3. Decomposition of $\mathrm{BuD}$ in $\mathrm{MSD}(2.0 \mathrm{M})$-cyclohexane $(4.7 \mathrm{M})$ at $140^{\circ} \mathrm{C}$

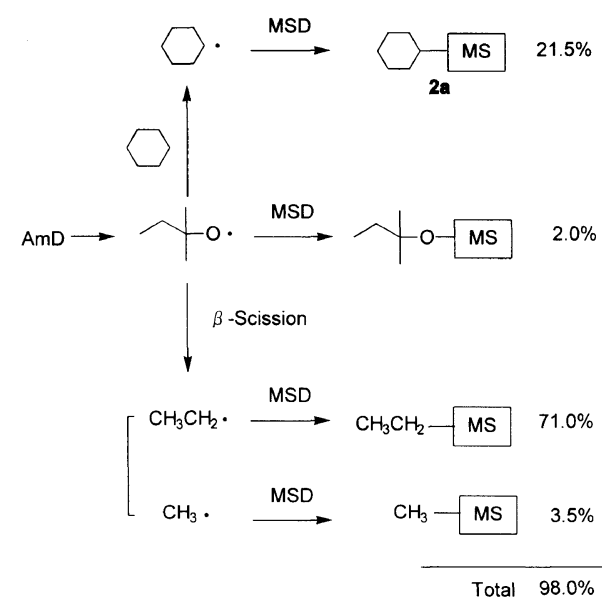

Scheme 4. Decomposition of AmD in MSD (2.0 M)-cyclohexane $(4.7 \mathrm{M})$ at $140^{\circ} \mathrm{C}$. tion by $t$-butoxy radicals from cyclohexane $\left(1.6 \times 10^{6}\right.$ $\left.\mathrm{M}^{-1} \mathrm{~s}^{-1}\right)$ is reported comparable to that for addition to styrene $\left(9.1 \times 10^{6} \mathrm{M}^{-1} \mathrm{~s}^{-1}\right)$ at $24^{\circ} \mathrm{C} .{ }^{5}$ Thus, the present results are consistent with those reported.

In a previous work, ${ }^{2}$ we studied the reactions of alkoxy radicals in neat $\mathrm{MSD}$ at $140^{\circ} \mathrm{C}$. Typical reactions were addition, $\beta$-scission, and 1,5-hydrogen shift (1,5-H shift, only in the case of $t$-hexyloxy radicals). The same reactions were also observed in this study. The distribution (normalized to $100 \%$ ) of these reactions and $\mathrm{H}$-abstraction are summarized in Table I, including the results in neat MSD. H-Abstraction percentages were correlated with the addition or self-reactions $(\beta$-scission and $1,5-\mathrm{H}$

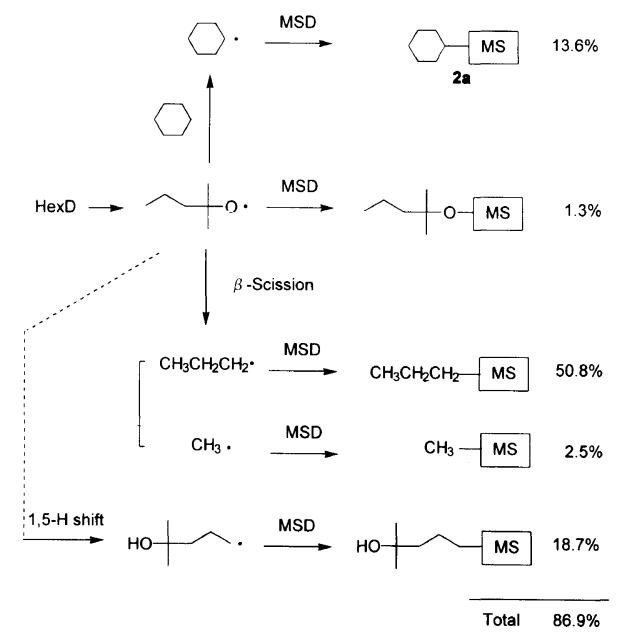

Scheme 5. Decomposition of HexD in MSD (2.0 M)-cyclohexane $(4.7 \mathrm{M})$ at $140^{\circ} \mathrm{C}$

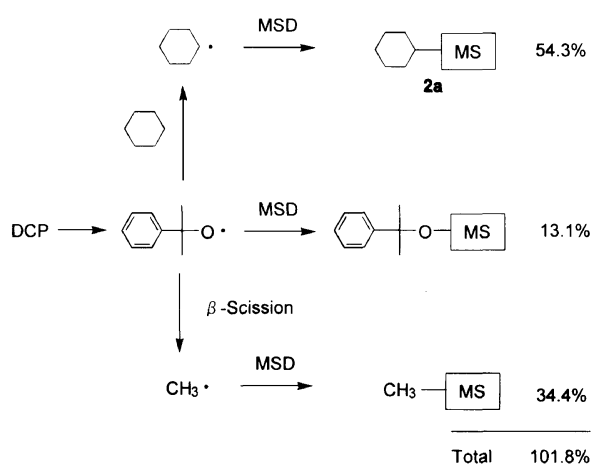

Scheme 6. Decomposition of DCP in MSD (2.0 M)-cyclohexane $(4.7 \mathrm{M})$ at $140^{\circ} \mathrm{C}$.

Table I. Distribution of reactions of alkoxy radicals at $140^{\circ} \mathrm{C}^{\mathrm{a}}$

\begin{tabular}{|c|c|c|c|c|c|c|c|}
\hline \multirow{2}{*}{ Alkoxy radical ${ }^{\mathrm{b}}$} & \multirow{2}{*}{$\frac{[\mathrm{MSD}]}{\mathrm{M}}$} & \multirow{2}{*}{$\frac{[\mathrm{CyH}]^{\mathrm{c}}}{\mathrm{M}}$} & \multirow{2}{*}{$\frac{\text { Addition }}{\%}$} & \multicolumn{2}{|c|}{$\beta$-Scission $/ \%$} & \multirow{2}{*}{$\frac{1,5-\text { Shift }}{\%}$} & \multirow{2}{*}{$\frac{\text { H-Abstraction }}{\mathrm{d}}$} \\
\hline & & & & $\mathrm{R}$ & $\mathrm{Me}$ & & \\
\hline \multirow[t]{2}{*}{$t-\mathrm{BuO} \cdot(\mathrm{R}=\mathrm{Me})$} & 4.2 & 0 & 62 & 38 & - & - & - \\
\hline & 2.0 & 4.7 & 10 & 11 & - & - & $79(72)^{\mathrm{e}}$ \\
\hline \multirow[t]{2}{*}{$t-\mathrm{AmO} \cdot(\mathrm{R}=\mathrm{Et})$} & 4.2 & 0 & 5 & 90 & 5 & - & - \\
\hline & 2.0 & 4.7 & 2 & 72 & 4 & - & $22(22)^{\mathrm{e}}$ \\
\hline \multirow[t]{2}{*}{$t$-HexO $\cdot(\mathrm{R}=\mathrm{Pr})$} & 4.2 & 0 & 3 & 72 & 6 & 19 & - \\
\hline & 2.0 & 4.7 & 1 & 58 & 3 & 22 & $16(14)^{\mathrm{e}}$ \\
\hline \multirow[t]{2}{*}{$\mathrm{CuO} \cdot(\mathrm{R}=\mathrm{Ph})$} & 4.2 & 0 & 41 & $<1$ & 59 & - & - \\
\hline & 2.0 & 4.7 & 13 & $<1$ & 34 & - & $53(54)^{\mathrm{e}}$ \\
\hline
\end{tabular}

${ }^{\mathrm{a}}$ Normalized to $100 \%$. ${ }^{\mathrm{b}}$ Alkoxy radicals from $2 .{ }^{\mathrm{c}}$ Cyclohexane. ${ }^{\mathrm{d}}$ Hydrogen abstraction from cyclohexane. ${ }^{\mathrm{e}}$ Absolute yields. 
Table II. Yields of dicyclohexyl from the decomposition of $\mathbf{3}$ in cyclohexane at $150^{\circ} \mathrm{C}^{\mathrm{a}}$

\begin{tabular}{cc}
\hline & Dicyclohexyl $^{\mathrm{b}}$ \\
\cline { 2 - 2 } Peroxide & $\%$ \\
\hline BuD & 42 \\
AmD & 40 \\
HexD & 44 \\
DCP & 48 \\
\hline
\end{tabular}

${ }^{\mathrm{a}}$ Initial peroxide concentration, $0.01 \mathrm{M}$; decomposition percentages, $>99.9 \%$. ${ }^{\mathrm{b}}$ Based on peroxides.

shift) obtained in neat MSD. H-Abstraction ability decreases with increasing rate of self-reactions giving alkyl radicals. $\mathrm{H}$-Abstraction ability of dialkyl peroxides is determined by the life time of alkoxy radicals produced. This is consistent with the fact that alkoxy radicals act as main or exclusive hydrogen abstractors under the present conditions (see above).

\section{Dimer Method}

The present results obtained by MSD trapping were compared with those by dimer method. The thermal decomposition of $\mathbf{3}$ was carried out in neat cyclohexane at $150^{\circ} \mathrm{C}$. The yields of cyclohexane dimer (dicyclohexyl) were determined by GLC. The results are shown in Table II. The dimer yields were almost the same for all peroxides $(40-50 \%)$. Bimolecular self-reactions of cyclohexyl radicals are known to involve coupling (eq 4a) and disproportionation (eq 4 b) at 48/52 (eq 4a/4b). ${ }^{6}$

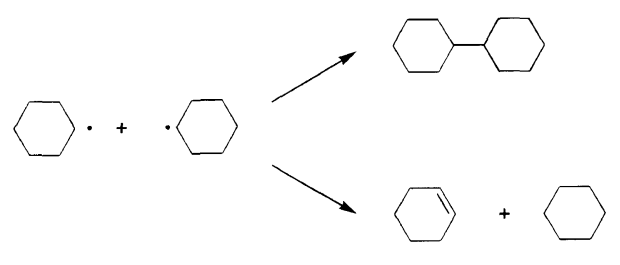

This means that the maximum dimer yield is about $50 \%$. The hydrogen abstraction ability of the dialkyl peroxides in neat cyclohexane may thus be independent of the peroxide structure and close to $100 \%$. Alkyl radicals as well as alkoxy radicals act as effective hydrogen abstractors in neat cyclohexane, because no alkyl radical trappers such as MSD are present in the reaction system.

\section{Summary}

MSD trapping is useful for investigating $\mathrm{H}$-abstraction ability of organic peroxides. To the best of our knowledge, this is the first study to show the large difference in $\mathrm{H}$-abstraction ability among dialkyl peroxides in the presence of styrenic compounds. The present results are applicable to the graft polymerization of styrene onto polyethylene since MSD and cyclohexane are model compounds of styrene and polyethylene, respectively. The graft efficiency may decrease in the order: $\mathrm{BuD}>\mathrm{DCP}>\mathrm{AmD}>\mathrm{HexD}$. Although HexD is not efficient in grafting reactions, the poor $\mathrm{H}$-abstraction ability should have the advantage of giving better controlled polymers (less branch and gel) in the usual polymerization of vinyl monomers. The advantage of MSD trapping over the dimer method is that the difference in $\mathrm{H}$-abstraction ability between alkoxy and alkyl radicals is distinctly differentiated. To know or predict $\mathrm{H}$-abstraction reaction in the presence of monomers such as styrene, we should use $\mathrm{H}$-abstraction data obtained by MSD trapping rather than those by the dimer method. Studies on $\mathrm{H}$-abstraction ability of various organic peroxides in different $\mathrm{H}$-donor substrates and effects of concentrations of MSD and H-donor substrates on $\mathrm{H}$ abstraction ability of peroxides are currently underway.

\section{REFERENCES}

1. J. D. Van Drumpt and H. H. J. Oosterwijk, J. Polym. Sci., Polym. Chem. Ed., 14, 1495 (1976).

2. Y. Watanabe, H. Ishigaki, H. Okada, and S. Suyama, Polym. J., 29, 366 (1997).

3. Y. Watanabe, H. Ishigaki, H. Okada, and S. Suyama, Chem. Lett., 1089 (1993); S. Suyama, H. Ishigaki, Y. Watanabe, and T. Nakamura, Polym. J., 27, 371 (1995); S. Suyama, H. Ishigaki, Y. Watanabe, and T. Nakamura, Polym. J., 27, 503 (1995).

4. F. Carrock and M. Szwarc, J. Am. Chem. Soc., 81, 4138 (1959).

5. J. Chateauneuf, J. Lusztyk, and K. U. Ingold, J. Am. Chem. Soc., 110, 2877 (1988); J. A. Howard and J. C. Scaiano, in "Landolt-Börnstein. New Series. Radical Reaction Rates in Liquids," Vol. 13d, H. Fischer, Ed., Springer-Verlag, Berlin, 1984, p 19.

6. W. A. Cramer, J. Phys. Chem., 71, 1171 (1967); K. U. Ingold, in "Free Radicals," Vol. I, J. K. Kochi, Ed., John Wiley \& Sons, New York, N.Y., 1973, p 51. 